\title{
High-Resolution Magnetic Force Microscopy Using Carbon Nanotube Probes Fabricated Directly by Microwave Plasma-Enhanced Chemical Vapor Deposition
}

\author{
Kei Tanaka, Masamichi Yoshimura, and Kazuyuki Ueda \\ Nano High-Tech Research Center, Toyota Technological Institute, 2-12-1 Hisakata, Tempaku-ku, Nagoya 468-8511, Japan \\ Correspondence should be addressed to Masamichi Yoshimura, yoshi@toyota-ti.ac.jp
}

Received 27 September 2008; Accepted 31 October 2008

Recommended by Rakesh Joshi

Carbon nanotubes (CNTs) have been successfully grown on the tip apex of an atomic force microscopy (AFM) cantilever by microwave plasma-enhanced chemical vapor deposition (MPECVD). Both scanning electron microscopy (SEM) and transmission electron microscopy (TEM) observations reveal that the diameter of the CNTs is $\sim 30 \mathrm{~nm}$ and the magnetic particles with diameter of $\sim 20 \mathrm{~nm}$, which was used as catalyst for the CNT growth, exist on the top. This CNT probe has been applied to magnetic force microscopy (MFM) on the ultrahigh-density magnetic recording media with 1200 kilo flux change per inch (kfci).

Copyright ( $) 2009$ Kei Tanaka et al. This is an open access article distributed under the Creative Commons Attribution License, which permits unrestricted use, distribution, and reproduction in any medium, provided the original work is properly cited.

\section{Introduction}

Magnetic force microscopy (MFM) is a powerful tool to study the magnetic domain structure at a microscopic level, and is widely used in the research and development of magnetic nanodevices. Images with $50 \mathrm{~nm}$ resolution are routinely obtained when using commercial microscope and magnetic probes. However, higher resolution becomes more desired with increased recording density in future magnetic recording media. It is well known that the MFM resolution is limited by the tip geometry and height above the sample surface [1]. In particular, decrease in tip diameter is the most significant factor to improve the resolution.

Thus to achieve reedy tips, methods using focused ion beam (FIB) $[2,3]$ and the utilization of carbon nanotube (CNT) [4-9] have been proposed. There have been two methods for the fabrication of CNT-based MFM probes: a mechanical attachment of metal-filled CNTs or catalystattached CNTs under scanning electron microscopic (SEM) observation [7-9], and a coating of magnetic metal over a preattached CNT probe [4-6]. In the latter technique, Kuramochi et al. reported that the magnetic recording media up to 1100 kilo flux changes per inch (kfci) has been clearly observed with a resolution of approximately $10 \mathrm{~nm}$
[4]. However, the methods described above require timeconsuming mechanical attachment or multiple processes, and seem inadequate for the large-scale production.

In a previous work, we successfully fabricated CNT probes for scanning tunneling microscopy (STM) by microwave plasma-enhanced chemical vapor deposition (MPECVD) [10]. This direct growth is quite suitable for the mass-scaled fabrication. It should be noted that the transmission electron microscopy (TEM) observation revealed the existence of magnetic particle as a catalyst on the top of each CNT. This feature can be applicable to MFM probes. Here, we have performed a growth of CNTs on the AFM cantilever made of $\mathrm{Si}$, and have evaluated the resolution in the MFM measurement of longitudinal magnetic recording media with 1200 kfci.

\section{Experimental}

The growth of CNTs on the $\mathrm{Si}$ cantilever was performed by using an MPECVD system (CVD-CN-100, Ulvac Japan, Ltd., Kanagawa, Japan). A Co thin film $20 \mathrm{~nm}$ thick, as the catalyst, was coated on the Si cantilever $(k=2.8 \mathrm{~N} / \mathrm{m})$ by vacuum deposition. It was then set into the metal holder with seat mesh on the small hole in order to avoid the electric 


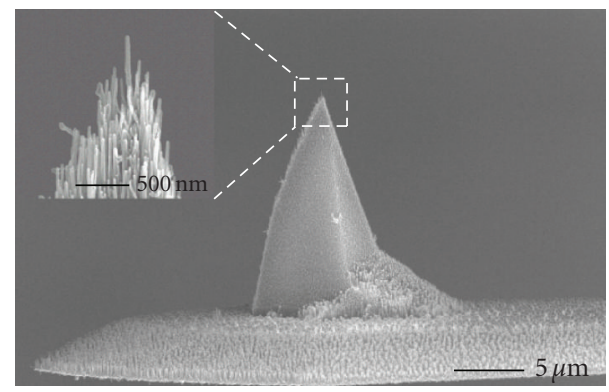

FIGURE 1: SEM image of CNTs on the tip apex of Si cantilever.

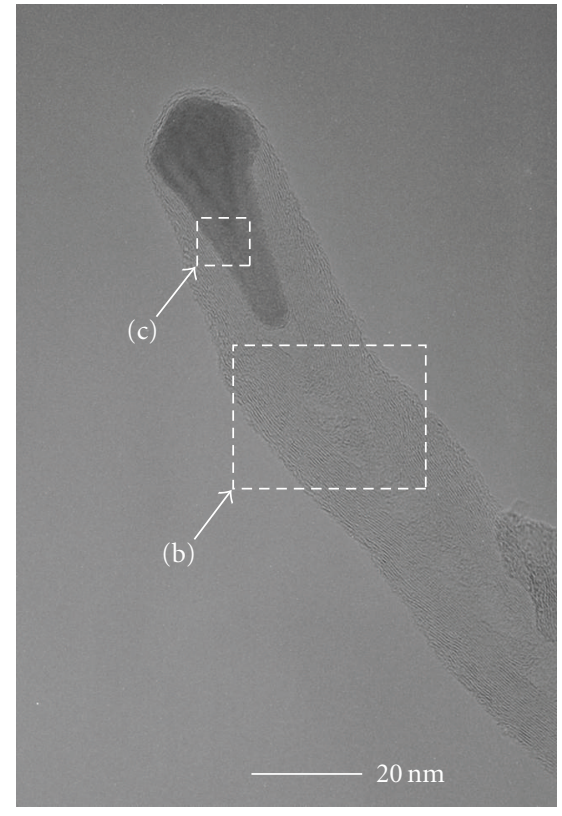

(a)

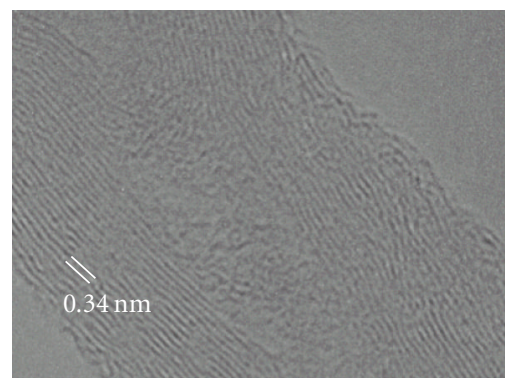

(b)

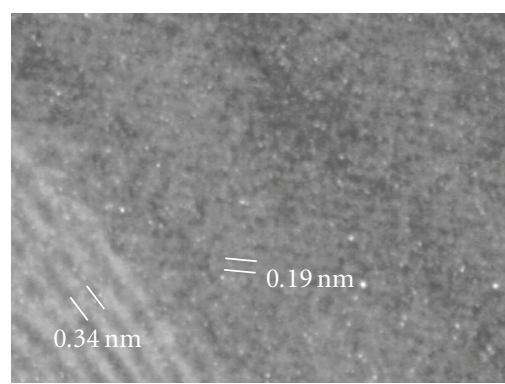

(c)

FIGURE 2: (a) TEM images of cocatalyzed CNT that scraped away from Si substrate. (b) and (c) are enlarged images of (a).

discharge and ion etching from the plasma [10], and was placed on the electrode in the MPECVD system. The mixed gas of $\mathrm{CH}_{4}$ and $\mathrm{H}_{2}$ was used for the CVD growth. The flow rate of $\mathrm{H}_{2}$ and $\mathrm{CH}_{4}$ was 80 and $20 \mathrm{sccm}$, respectively. Total gas pressure was set at $226 \mathrm{~Pa}$. The microwave used was $2.45 \mathrm{GHz}, 500 \mathrm{~W}$ and the growth time was 7 minutes. During the growth process, a voltage of $200 \mathrm{~V}$ was applied between the electrodes. Prior to the CNT growth, the cantilever was exposed to hydrogen plasma for cleaning as well as for activation of catalysts. During CVD process, no heater was used, but the substrate temperature was raised to approximately $600^{\circ} \mathrm{C}$ as measured by thermocouple. The morphology of tip apex after growth was observed by a field emission scanning electron microscope (FE-SEM, Hitachi, S4700). A high-resolution transmission electron microscope (TEM, JEOL, JEM2000EX) was used to determine the atomic structure of the CNT. In order to examine MFM performance, the surface of longitudinal magnetic recording media with densities of $600,800,1000$, and $1200 \mathrm{kfci}$ was observed by Nanoscpoe III (Digital Instruments, NY, USA) in air.

\section{Results and Discussion}

Figure 1 shows an SEM image of CNTs grown on the tip apex of the Si cantilever. The CNTs $\sim 30 \mathrm{~nm}$ in diameter are clearly observed at the tip apex. Figure 2 shows a TEM image of a typical CNT similarly grown on an Si wafer. The catalyst particle exists on the top of CNT. This suggests that CNTs grew via the so-called tip growth mechanism [11]. The CNT shows tubular structure with graphene layers (lattice spacing of $0.34 \mathrm{~nm}$ ) as resolved in the enlarged TEM image (Figure 2(b)). The magnified image of catalyst particle is shown in Figure 2(c), where the lattice of $0.19 \mathrm{~nm}$ is thoroughly observed. The lattice spacing corresponds to $\mathrm{Co}_{3} \mathrm{C}(103)$, suggesting that the $\mathrm{CNT}$ is grown with a nanoparticle of single crystalline $\mathrm{Co}_{3} \mathrm{C}$ at its top [12]. 


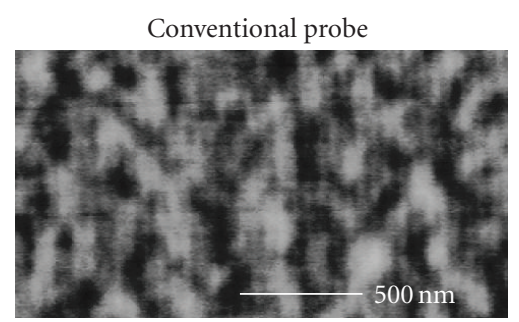

(a)

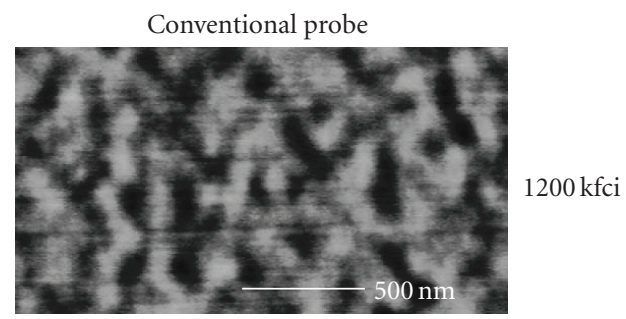

(c)

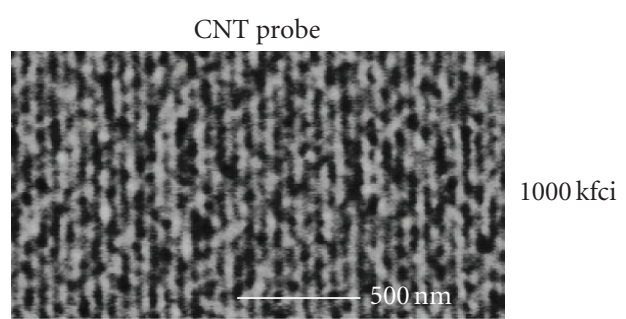

(b)

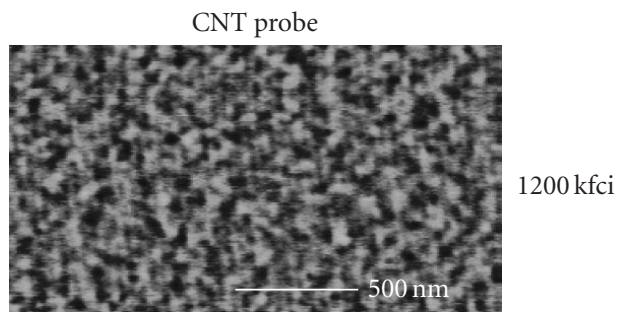

(d)

FIGURE 3: MFM images of ultrahigh-density recording media of 1000 and $1200 \mathrm{kfci}$ using conventional and CNT probes. (a), (c) show MFM image obtained by conventional probe. (b), (d) show MFM image obtained by CNT probe. The recording densities were (a) and (b) $1000 \mathrm{kfci}$, (c) and (d) $1200 \mathrm{kfci}$.

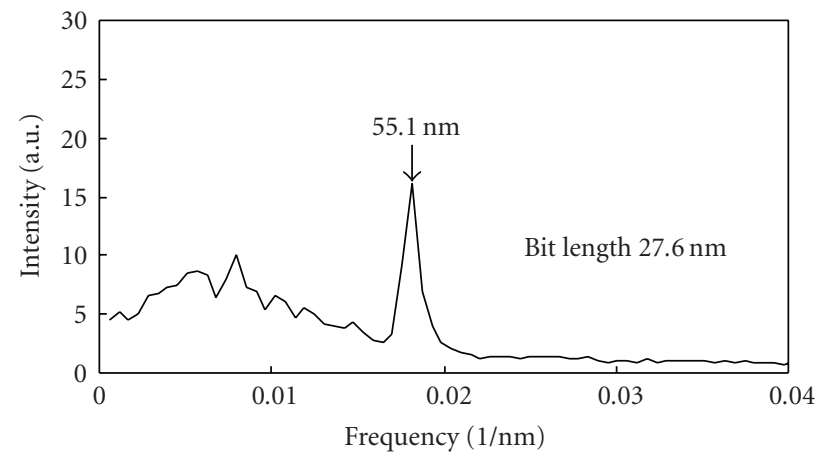

(a) $1000 \mathrm{kfci}$

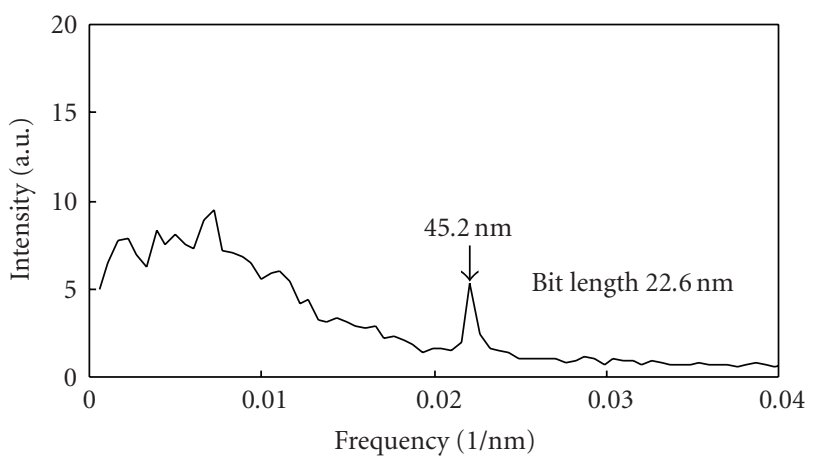

(b) $1200 \mathrm{kfci}$

FIGURE 4: Power spectra corresponding to Figures 3(b) and 3(d). We have averaged twenty power spectra after performing a Fourier transform on a single-line profile obtained from MFM image.

Using this fabricated CNT probe and a conventional probe, MFM observation is performed for the ultrahighdensity recording media of 1000 and $1200 \mathrm{kfci}$, as shown in Figure 3. In the case of conventional probe, a stripe pattern of magnetic domain structure is observed up to $1000 \mathrm{kfci}$ (Figure 3(a)), though the periodicity is not so clear. Irregular pattern appears appreciably comparable to the periodicity. In the case of $1200 \mathrm{kfci}$, no stripe magnetic domain structure is observed, as shown in Figure 3(c). In contrast, the recording period is clearly resolved up to $1000 \mathrm{kfci}$ using the present CNT probe (Figure 3(b)), and even for $1200 \mathrm{kfci}$, the periodicity is visible on the background of the irregular pattern due to the limitation in the resolution and/or sample preparation.

Figure 4 shows the power spectra corresponding to Figures $3(\mathrm{~b})$ and $3(\mathrm{~d})$. These spectra were obtained by averaging twenty spectra after performing a Fourier transform of a single-line profile of the cross-section in MFM image. As shown in Figure 4, peaks at 55.1 and $45.2 \mathrm{~nm}$ are recognized (which is double the recoding bit size). These values are larger than the calculated values of 50.8 and $43.2 \mathrm{~nm}$ for 1000 and $1200 \mathrm{kfci}$. This may be due to inaccuracies in the recording process [2]. In these power spectra, strong background is observed on the low-frequency side. The inverse Fourier analysis indicates that this is caused by an irregular period due to disorder of the longitudinal recording. The writing of $1200 \mathrm{kfci}$ is known to be difficult in the case of the longitudinal recording.

In order to estimate attainable resolution, we follow analysis procedure reported by Kuramochi et al. [4]. Figure 5(a) shows normalized intensity of power spectrum as a function of recording density. It is observed that the intensity decreases with increase in recording density. The intensity decreases with the increase of recording density. 


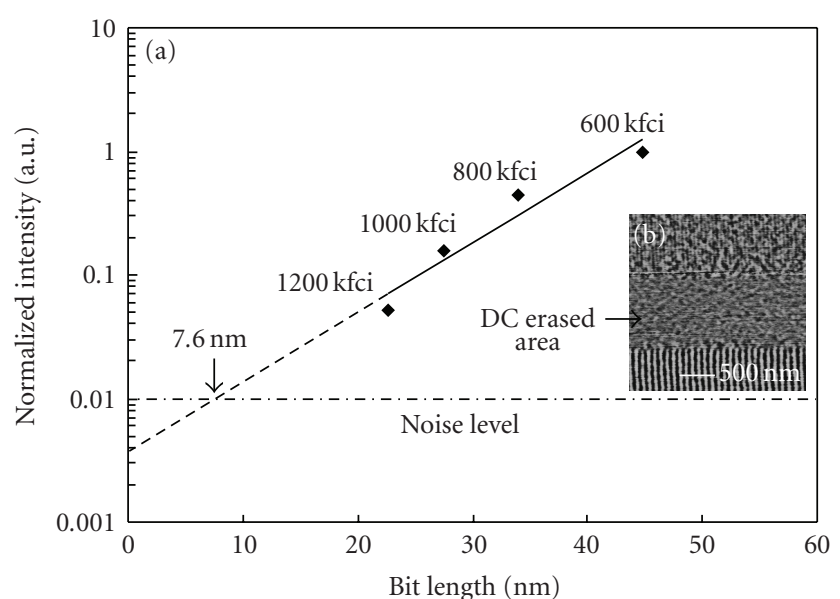

FIGURE 5: Plot of normalized intensity of power spectrum at the various recording bit length. The solid line was obtained by linear fitting for experimental plot. The sloping dashed line was obtained by extrapolating of solid line. The noise level was obtained from DC-erased area in (b) MFM image.

The attainable resolution in the observation of recording media is obtained from intersecting point of extrapolation of solid line and noise level line in Figure 5 [4]. The noise level was obtained from DC-erased area in MFM image as shown in Figure 5(b). The intersecting point corresponding to attainable resolution is around $7.6 \mathrm{~nm}$, which is comparable to the value reported in [4]. It should be noted that in spite of the CNT-MFM probe by the present simple fabrication process without coating of magnetic materials, the resolution has been improved compared with conventional probe. The mass-scale fabrication of high-resolution CNT-MFM probes is also expected by this direct growth method.

\section{Conclusion}

In conclusion, we have successfully fabricated the CNT probes for MFM by direct growth using MPECVD. The CNTs of $\sim 30 \mathrm{~nm}$ in diameter have been grown on the tip apex of Si cantilever, and the magnetic particle $(\sim 20 \mathrm{~nm})$ is located at the end. The MFM observation using the fabricated CNT probe for magnetic recording media is demonstrated. The CNT probe resolves a stripe magnetic domain structure of ultrahigh-density recording media of 1000 and $1200 \mathrm{kfci}$ much clearer than the conventional probe.

\section{Acknowledgments}

The authors would like to thank Dr. A. Okamoto (Toyota Central R\&D Lab., Inc., Aichi, Japan) for his help in TEM observation, Dr. M. Oshiki (Fujitsu Ltd.) and Dr. I. Okamoto (Yamagata Fujitsu Ltd., Yamagata, Japan) for supplying the ultrahigh-density magnetic recording media. This work is supported by the "High-Tech Research Center" project for Private Universities: matching fund subsidy from the Ministry of Education, Culture, Sports, Science and Technology (MEXT), 2006-2008.

\section{References}

[1] D. Rugar, H. J. Mamin, P. Guethner, et al., "Magnetic force microscopy: general principles and application to longitudinal recording media," Journal of Applied Physics, vol. 68, no. 3, pp. 1169-1183, 1990.

[2] L. Gao, L. P. Yue, T. Yokota, et al., "Focused ion beam milled CoPt magnetic force microscopy tips for high resolution domain images," IEEE Transactions on Magnetics, vol. 40, no. 4, pp. 2194-2196, 2004.

[3] G. N. Phillips, M. Siekman, L. Abelmann, and J. C. Lodder, "High resolution magnetic force microscopy using focused ion beam modified tips," Applied Physics Letters, vol. 81, no. 5, pp. 865-867, 2002.

[4] H. Kuramochi, T. Uzumaki, M. Yasutake, A. Tanaka, H. Akinaga, and H. Yokoyama, "A magnetic force microscope using CoFe-coated carbon nanotube probes," Nanotechnology, vol. 16, no. 1, pp. 24-27, 2005.

[5] H. Kuramochi, H. Akinaga, Y. Semba, et al., "CoFe-coated carbon nanotube probes for magnetic force microscope," Japanese Journal of Applied Physics, vol. 44, no. 4A, part 1, pp. 2077-2080, 2005.

[6] Z. Deng, E. Yenilmez, J. Leu, et al., "Metal-coated carbon nanotube tips for magnetic force microscopy," Applied Physics Letters, vol. 85, no. 25, pp. 6263-6265, 2004.

[7] A. Winkler, T. Mühl, S. Menzel, et al., "Magnetic force microscopy sensors using iron-filled carbon nanotubes," Journal of Applied Physics, vol. 99, no. 10, Article ID 104905, 5 pages, 2006.

[8] T. Arie, H. Nishijima, S. Akita, and Y. Nakayama, "Carbonnanotube probe equipped magnetic force microscope," Journal of Vacuum Science and Technology B, vol. 18, no. 1, pp. 104-106, 2000.

[9] N. Yoshida, M. Yasutake, T. Arie, S. Akita, and Y. Nakayama, "Quantitative analysis of the magnetic properties of metalcapped carbon nanotube probe," Japanese Journal of Applied Physics, vol. 41, no. 7B, pp. 5013-5016, 2002.

[10] K. Tanaka, M. Yoshimura, and K. Ueda, "Fabrication of carbon nanotube tips for scanning tunneling microscopy by direct growth using the microwave plasma-enhanced chemical vapor deposition," e-Journal of Surface Science and Nanotechnology, vol. 4, pp. 276-279, 2006.

[11] R. T. K. Baker, M. A. Barber, P. S. Harris, F. S. Feates, and R. J. Waite, "Nucleation and growth of carbon deposits from the nickel catalyzed decomposition of acetylene," Journal of Catalysis, vol. 26, no. 1, pp. 51-62, 1972.

[12] M. Tanemura, K. Iwata, K. Takahashi, et al., "Growth of aligned carbon nanotubes by plasma-enhanced chemical vapor deposition: optimization of growth parameters," Journal of Applied Physics, vol. 90, no. 3, pp. 1529-1533, 2001. 

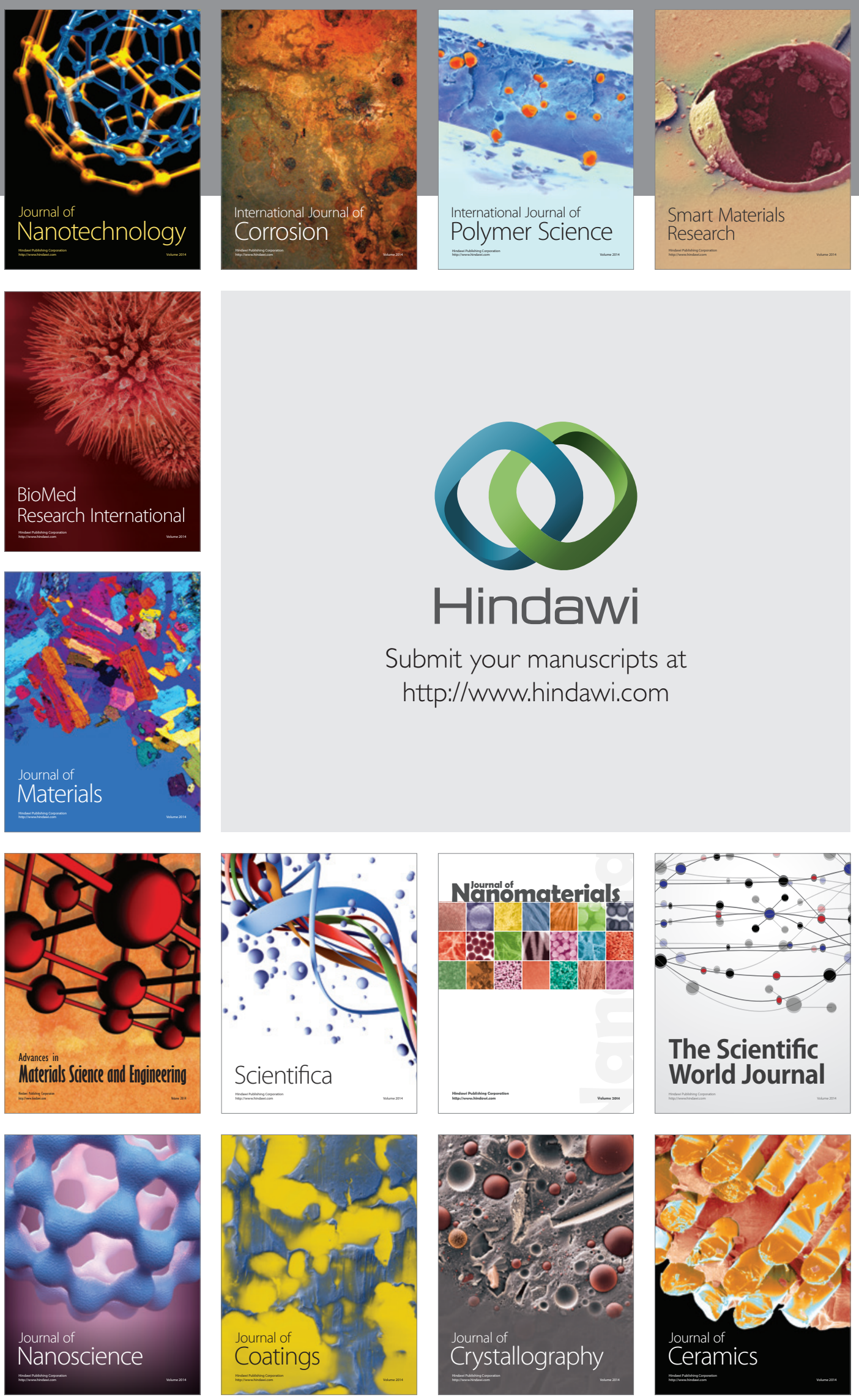

The Scientific World Journal

Submit your manuscripts at

http://www.hindawi.com

\section{World Journal}

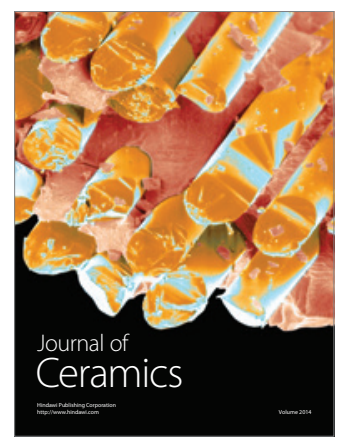

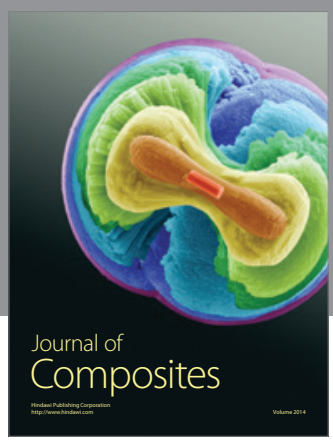
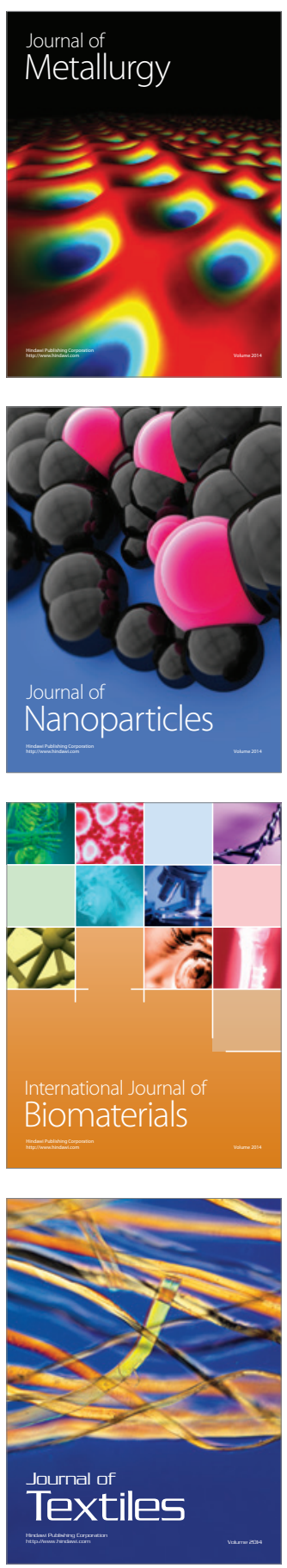